\title{
EVALUATION OF OUTCOME OF MEDIAL OPENING WEDGE HIGH TIBIAL OSTEOTOMY FOR UNICOMPARTMENTAL KNEE OSTEOARTHRITIS
}

\section{Orthopaedics}

Dr. R. N. Shewale Professor, Department of Orthopedics, M.G.M. Medical College Aurangabad.

Dr. Ketan J. Khatri*
Resident, Department of Orthopedics, M.G.M. Medical College Aurangabad. *Corresponding Author

\begin{abstract}
Background: Osteoarthritis is often found in weight-bearing joints, the knee being the most common site. Most patients of symptomatic osteoarthritis of knee are associated with varus malalignment that is causative or contributory to painful arthritis. Correcting the malalignment of the knee relieves symptoms by transferring the functional load to the unaffected compartment. The goal of the treatment is to relieve medial compartment knee pain and slow down the arthritic progression. We report the outcome of a simple technique of medial opening wedge high tibial osteotomy in treating the medial compartment osteoarthritis of the knee.

Materials And Method: A prospective interventional study was carried out over a period of 2 years from November 2017 to October 2019 in a sample size of randomly selected 41 patients having unilateral knee osteoarthritis. All patients after a proper preoperative assessment underwent high tibial osteotomy and the outcome was evaluated using knee society scoring system.

Results: The age of the patients ranged from $40-65$ years with a mean age of 51 years, 13 were males and 28 were females showing female predominance $(68.2 \%)$. The mean knee score and the mean functional score of the patients before surgery were 60 and 61.95 respectively and post operatively at the end of 6 months the knee score and functional score was 77 and 80.53 respectively. By the end of 6 months out of 41 patients, 12 patients (29.2\%) had excellent functional outcome, 18 patients $(43.90 \%)$ had good functional outcome, 09 patients $(21.95 \%)$ had fair functional outcome while only 02 patients $(04.87 \%)$ had poor functional outcome.

Conclusions: The present study shows that HTO is a good option in isolated medial compartment OA of knee. Significant increase in the knee score and functional score was found after high tibial osteotomy for the patients of osteoarthritis with varus deformity. Success of high tibial osteotomy relies on appropriate patient selection, proper osteotomy type and precise surgical technique.
\end{abstract}

\section{KEYWORDS}

High tibial osteotomy, open wedge, genu varum, knee osteoarthritis.

\section{INTRODUCTION}

Knee osteoarthritis is much more prevalent in India than in west and accounts as much more disability as any of other chronic conditions. Osteoarthritis once considered being a disease of elderly but in the recent years it is becoming more common even in people aged less than 50 years ${ }^{[1]}$. Many patients present with Unicompartmental disease, and the medial compartment is almost 10 times more frequently involved than the lateral compartment ${ }^{[2]}$. Most patients with OA knee have varus malalignment where the mechanical axis and load bearing axis passes through the medial compartment. The initial treatment of symptomatic medial Unicompartmental knee OA is non-operative, consisting of patient education (weight reduction), medication and if needed physical therapy. When non-operative treatment fails high tibial valgus osteotomy (HTO) is an accepted treatment of medial Unicompartmental OA of the knee with varus alignment, especially in younger active patients ${ }^{[3]}$. Corrective osteotomy is done to transfer the load bearing from the pathologic to the normal compartment of the knee. A successful outcome of the osteotomy relies on proper patient selection, and achievement and maintenance of adequate operative correction ${ }^{[4]}$. Medial opening wedge high tibial osteotomy (HTO) is a biomechanical intervention designed to alter dynamic knee joint loading, with the aim of improving patient function and decreasing pain.

\section{AIMS AND OBJECTIVE}

Aim of the study was to evaluate the functional outcome among patients undergoing medial opening wedge high tibial osteotomy for Unicompartmental knee osteoarthritis with varus deformity.

Objective of the study was to assess the deformity correction by X-ray, Scannogram and clinical examination and the relief of symptoms of osteoarthritis, function and patient satisfaction using Knee Society scoring system.

\section{MATERIALS AND METHODS}

A prospective interventional study was conducted in the Dept. of orthopedics, at our College for 2 years from November 2017 to October 2019 involving randomly selected 41 patients.

Patients of age group 40-65 yrs with moderate Unicompartmental OA and with varus deformity $\leq 20^{\circ}$ and having range of motion of more than $90^{\circ}$ were included in study.

The patients with bi compartmental and tri compartmental osteoarthritis having restricted range of movements at knee or flexion contracture of more than 15 degrees and patients with previous history of knee surgery were excluded from the study. Clearance from the Ethical Committee of the institute and permission was obtained prior to initiation of the study. A written informed consent was obtained from the patients who agreed for operative treatment and to be the part of our study.

\section{Preoperative Planning}

The radiographic grade of osteoarthritis was scored according to Ahlbäck (1968) ${ }^{[5]}$ and measured on standard short posteroanterior radiographs in standing position. Mechanical axis from the center of the femoral head to the center of the knee, anatomical axis from the piriformis fossa to the center of the knee joint and a line through the long axis of the tibia, and weight bearing axis from the center of the femoral head to the center of ankle join) were calculated for determining the location and amount of corrective osteotomy.(Fig. 1).

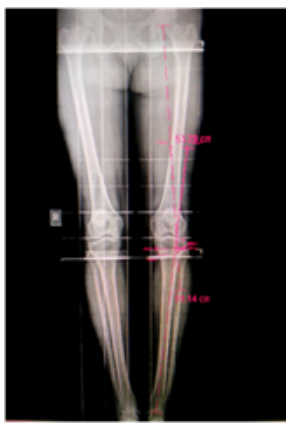

Fig 1: Preoperative Scannogram Showing Marking Of Axis And Angle Of Osteotomy.

\section{Operative Procedure}

Patient was supine on a radiolucent operating table and tourniquet was applied to thigh. The anatomical landmarks were drawn over the skin and a 5 -cm vertical skin incision was made over the center between the tibial tuberosity and the posteromedial border of the tibia. The subcutaneous tissue was dissected and the pes tendons were retracted to expose the superficial medial collateral ligament. Two guide wires were inserted parallel at a point $3.5-4 \mathrm{~cm}$ below the medial joint line and passed obliquely towards the tip of the fibular head to mark the direction of the osteotomy. Tibial osteotomy was performed immediately below the guide wires using an oscillating saw or an osteotome. The osteotomy Site was opened slowly to prevent 
fracturing of the lateral cortex. After achieving the planned width, an arthrodesis spreader was placed in the posteromedial corner of the osteotomy.

The 'cable technique' was used for the determination of malalignment measurements. Under image intensification a long measuring cable was placed at the center of the femoral head and at the center of the upper ankle joint. The axis was adjusted by opening or closing the bone spreader as required.

The T-buttress plate with its preassembled proximal drill sleeve was slid into a subcutaneous tunnel (Fig. 2). A temporary lag screw was inserted into the first plate hole distal to the osteotomy to compresses the lateral hinge by pulling the distal osteotomy segment towards the plate. The lag screw distal to the osteotomy gap was replaced by a bicortical locking screw and a locking head screw was inserted proximal of the gap. In the cases where width exceeded $13 \mathrm{~mm}$, autogenous cancellous bone graft harvested from the anterior iliac crest was transplanted in the osteotomy gap.
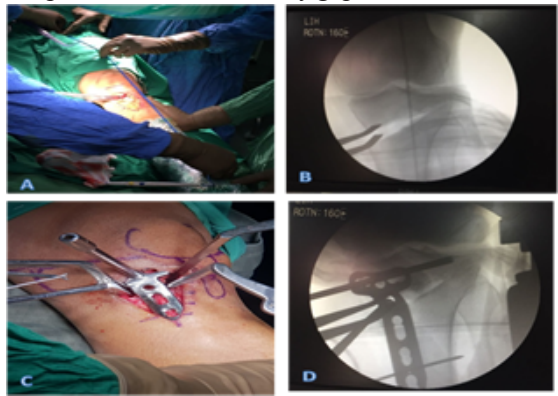

Fig 2: Intra Operative Images Of Surgical Procedure.

Fluoroscopy images of the knee in two planes were taken for documentation before the subcutaneous tissue and the skin closure while ensuring sufficient implant cover. Crepe bandage along with Long knee brace in full extension was applied to the leg post operatively (Fig. 3).
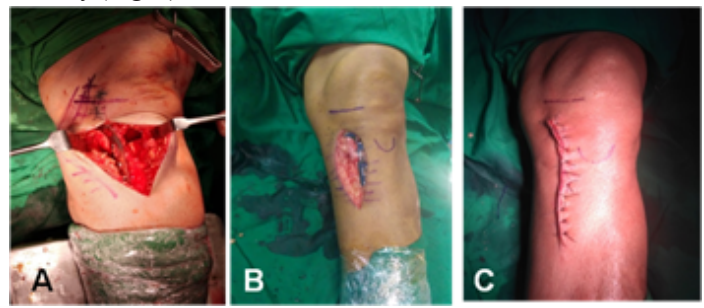

Fig 3: A) osteotomy site fixed with plate B) final position of plate C) closure of surgical site.

\section{Postoperative Protocol}

The limb was kept elevated in full extension with Long knee brace application. Patients were kept non weight-bearing for 8 weeks and later were allowed to walk without crutches according to pain and tolerance. X-rays were done immediate post operatively and were repeated at 6 weeks, 3 months and 6 months post surgery (Fig. 4).
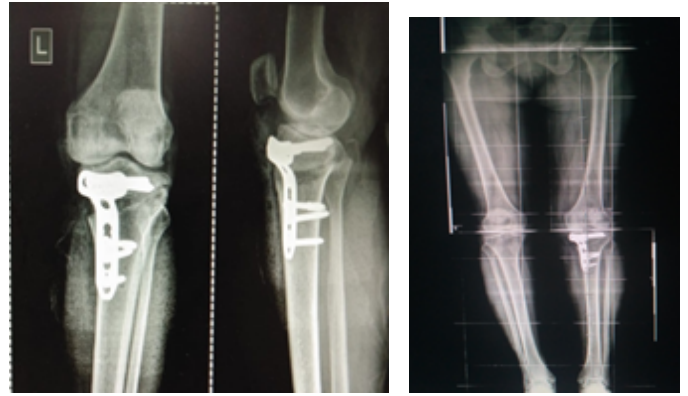

Fig 4: Post Operative X-ray Showing Fixation Of Osteotomy Site And Correction Of Axis.

Follow Up

Patients were routinely followed up at 6 weeks, 3 months and 6 months

(Fig. 5). Post surgery and evaluated clinically using knee society score (KSS) scoring system.
Score between

$100-80$ points were considered excellent

$79-70$ points were considered good

$69-60$ points were considered fair

$<60$ points were considered poor results

Patients were also evaluated signs of radiological union and alignment at every follow up. We used the Statistical Package for Social Science (SPSS) version21 for performing the statistical analyses. Mean, Standard Deviation \& Chi-square test were used to analyze the result and test the significance. Data were expressed as mean values \pm standard deviations (SD) for continuous variables. The p-value of $<$ 0.05 was considered to be statistically significant.

\section{RESULTS}

The age of the patients ranged from $40-65$ years with a mean of 51 years. Among the 41 patients 13 were males and 28 were females. Preoperatively, the mean knee society knee score was 60 with standard deviation of 9.45(Table. 1) and the mean knee society function score was 61.95 with standard deviation of 8.42 (Table. 3) in 41 patients of the study. Both the mean scores were considered as fair based on the categorization of the KSS scores. The postoperative mean knee score and functional score at 6 months was 77 and 80.53 respectively. The mean difference between preoperative and post operative comparison score showed statistically significant improvement in both the knee society score of 17 (Table. 2) and the functional score of 5.12 (Table. 4).

Table 1: Distribution of KSS knee Score:

\begin{tabular}{|l|l|l|}
\hline & KSS knee score \\
\hline & Mean & SD \\
\hline Preoperative & 60 & 9.45 \\
\hline 6 weeks & 67.5 & 8.45 \\
\hline 3 month & 73 & 9.75 \\
\hline 6 month & 77 & 10.69 \\
\hline
\end{tabular}

Table 2: Comparison of mean difference between pre-op, 6 weeks and 6 months of KSS knee score

\begin{tabular}{|l|l|l|l|}
\hline & Mean diff & t-value & p-value \\
\hline Pre-op vs. 6 weeks & 7.5 & 3.78 & $\mathrm{P}=0.003 \mathrm{~S}$ \\
\hline Pre-op vs. 3 months & 13 & 6.13 & $\mathrm{P}=0.001 \mathrm{~S}$ \\
\hline Pre-op vs. 6 months & 17 & 8.01 & $\mathrm{P}=0.001 \mathrm{~S}$ \\
\hline
\end{tabular}

By using unpaired t-test, $\mathrm{p}$-value $<0.05$ therefore there is significant difference between KSS knee score at pre operative vs. 6 week, pre operative vs. 3 month and pre operative vs. 6 month.

Table 3: Distribution of KSS Function Score:

\begin{tabular}{|l|l|l|}
\hline & KSS function score \\
\hline & Mean & SD \\
\hline Preoperative & 61.95 & 8.42 \\
\hline 6weeks & 70.53 & 8.27 \\
\hline 3 month & 75.41 & 8.38 \\
\hline 6 month & 80.53 & 8.66 \\
\hline
\end{tabular}

Table 4: Comparison Of Mean Difference Between Pre-op, 6 Weeks And 6 Months Of KSS Function Score

\begin{tabular}{|l|l|l|l|}
\hline & Mean diff & t-value & p-value \\
\hline Pre-op vs. 6 weeks & 8.58 & 4.65 & $0.001 \mathrm{~S}$ \\
\hline Pre-op vs. 3 months & 4.88 & 7.30 & $0.001 \mathrm{~S}$ \\
\hline Pre-op vs. 6 months & 5.12 & 10.01 & $0.001 \mathrm{~S}$ \\
\hline
\end{tabular}

By using unpaired t-test , $\mathrm{p}$-value $<0.05$ therefore there is significant difference between KSS knee score at pre operative vs. 6 week, pre operative vs. 3 month and pre operative vs. 6 month.(chart.1)

By using unpaired t-test, $\mathrm{p}$-value $<0.05$ therefore there is significant difference between KSS function score at pre operative vs. 6 week, pre operative vs. 3 month and pre operative vs. 6 month.(chart.2)

$31 \%$ of the patients had grade II type of Osteoarthritis and $42 \%$ had grade III type of Osteoarthritis. Low grade OA showed significant outcome (Table. 5)

Table 5: Final KSS Outcome According To Grade of OA.

\begin{tabular}{|l|l|l|l|l|l|l|}
\hline Grade & \multicolumn{3}{|l|}{ 6 MONTH KSS } & Total & $\begin{array}{l}\text { Chi } \\
\text { Of OA }\end{array}$ \\
\cline { 2 - 5 } & $\begin{array}{l}\text { Poor } \\
<\mathbf{6 0}\end{array}$ & $\begin{array}{l}\text { Fair } \\
\mathbf{6 0 - 7 0}\end{array}$ & $\begin{array}{l}\text { Good } \\
\mathbf{7 0 - 8 0}\end{array}$ & $\begin{array}{l}\text { Excellent } \\
\mathbf{8 0 - 1 0 0}\end{array}$ & & \\
\hline Grade-I & 00 & 01 & 00 & 04 & 05 & Chi- \\
\hline
\end{tabular}

International Journal of Scientific Research $H_{55} 55$ 


\begin{tabular}{|l|l|l|l|l|l|l|}
\hline Grade-II & 00 & 00 & 07 & 06 & 13 & square \\
\hline Grade-III & 02 & 05 & 08 & 02 & 17 & $=20.0$ \\
\hline Grade-IV & 00 & 03 & 03 & 00 & 06 & $\mathrm{P}=$ \\
\hline Total & 02 & 09 & 18 & 12 & 41 & $0.018 \mathbf{S}$ \\
\hline
\end{tabular}

By using chi square test, the final KSS outcome according to Grade of OA was significant with P value 0.018

Osteotomy size was 5-15 mm (mean, $8.4 \mathrm{~mm}$ ). Follow-up was 3-24 months with a mean of 1 year. Overall, 38 of the 41 HTOs included in this study united during the follow-up period (92.6\%). The average time to radiographic union was 14.8 weeks (range, 8 -24 weeks).

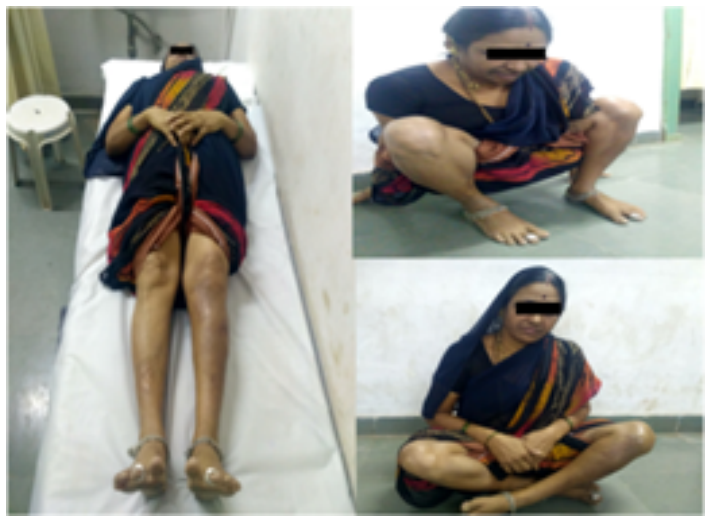

Fig 5: Clinical \& functional outcome of HTO done on left knee after 1 year.

\section{Complications:}

Two patients had superficial infection and two patients had hardware prominence causing pain. The patients with infection were treated with appropriate antibiotics and regular dressings. Subsequently they were followed up. Two patients had hardware prominence causing anterior leg pain and the implant was removed in them after 6 months.

\section{DISCUSSION:}

The results of valgus producing tibial osteotomy for knee osteoarthritis have been extensively reported in the literature. In the active patient with a life expectancy of 20 years or more a high tibial valgus producing osteotomy (HTO) is a generally accepted treatment that can result in excellent pain relief and function improvement with less pain in $80 \%$ of the time after five years which would, however, deteriorate with time ${ }^{[6]}$. It is rational to correct malalignment which would transfer the load to the less affected compartment of the knee to relieve clinical symptoms and hopefully slow down the progression of degeneration

AGE: - The age of the patients in our study ranged from 40 - 65 years with a mean age of 51 years. $75 \%$ of the patients were less than 55 years of age. By using chi square test, the final KSS outcome according to age was significant with $P$ value 0.046 . Based on our findings, the current overall role of osteotomies is clearly minor in treatment of patients with knee OA aged more than 60 years. Similar to our study, Bonasia et al. ${ }^{[8]}$ on studying 99 OWHTOs found that age was a preoperative variable significantly related to a poor outcome.

GENDER: - in our study, among the 41 patients 13 were males and 28 were females with male: female ratio of 1:2.1. Similar to our study, Hawker et al ${ }^{[9]}$ found that Knee OA was more common among the female population, with female patients generally suffering worse symptoms and greater disability than male. Also, Kumar et al ${ }^{[10]}$ studied 60 patients among which 37 were females and 23 were males showing predominance of females for knee OA.

GRADE OF OA: - in our study, $31 \%$ of the patients had grade II type of Osteoarthritis and $42 \%$ had grade III type of Osteoarthritis. It is generally agreed in the literature that a low degree of arthritis is linked to better outcomes; on the other hand, it is clear that Tri-compartmental $\mathrm{OA}$ is a contraindication to osteotomy ${ }^{[11]}$.

BMI: - BMI range in our study was 17-32 with average BMI of 23.05 patients were underweight, 24 had Normal BMI, 09 were overweight and 03 were obese. By using chi square test, the final KSS outcome according to BMI was significant with $P$ value 0.017

Our findings in the study co relate with the following studies. Flecher et al. ${ }^{[12]}$ studied 313 patients and found that those with a BMI of less than 30 had better outcomes. This finding was confirmed by Howells et al. ${ }^{[13]}$ in their study of 95 HTO patients. Bonasia et al. ${ }^{[8]}$, studying 99 OWHTOs, concluded that in patients with a BMI greater than 30 the risk of unsuccessful surgery is 10 times higher. On the other hand, Naudie et al. ${ }^{[14]}$, in a survival analysis of 106 HTOs, found that patients with a BMI lower than 25 have even worse results.

FUNCTIONAL OUTCOME:- Patients were analyzed for any complications and their functional outcome was compared with their previous status.

Preoperatively, the mean knee society knee score was 60 with standard deviation of 9.45 and the mean knee society function score was 61.95 with standard deviation of 8.42 in 41 patients of the study. Both the mean scores were considered as fair based on the categorization of the KSS scores. At 6 months post operative, the mean knee society knee score was 77 with standard deviation of 10.69 and the mean knee society function score was 80.53 with standard deviation of 8.66 . The mean difference of knee score and function score between preoperative and postoperative at 6 months was 17 and 5.12 respectively which was found to be statistically significant $(\mathrm{p}<0.001)$. Similar results were found the study conducted by Jung et al ${ }^{[15]}$. They found significant improvement in the mean Knee Society knee score from $68.5 \pm 11.9$ before the operation to $92.5 \pm 7.1$ at follow-up 2 years postoperatively. The mean Knee Society function score improved significantly from $62.3 \pm 11.4$ before the operation to $90.4 \pm 9.0$ at follow-up 2 years postoperatively.

In our study, by the end of 6 months out of 41 patients, 12 patients $(29.2 \%)$ had excellent functional outcome, 18 patients $(43.90 \%)$ had good functional outcome, 09 patients $(21.95 \%)$ had fair functional outcome while only 02 patients $(04.87 \%)$ had poor functional outcome. Pipino et $\mathrm{al}^{[16]}$ found increase in the mean KSS Functional score from 65 points preoperatively to 89 points at final follow-up ( $\mathrm{p}<$ $0.01)$. Good or excellent results were obtained in 106 patients $(94 \%)$. Seven patients (6\%) gave fair results, while none had a poor result.

OSTEOTOMY GAP \& BONE GRAFTING:- In our study, Osteotomy size range was 5-15 $\mathrm{mm}$ (mean, $8.4 \mathrm{~mm}$ )bone grafting was done in 15 patients in whom the osteotomy gap was more than $12 \mathrm{~mm}$. The average time to radiographic union was 14.8 weeks (range, 8-24 weeks). Kolb et al. ${ }^{[17]}$ analyzed 51 medial open wedge osteotomies and found that 50 osteotomies healed in an average period of 3 months without bone grafts and had excellent grading in $57 \%$, good in $24 \%$ patients by one rating system and $18 \%$ excellent, $63 \%$ good by another rating system.

\section{CONCLUSION}

In this study, there was significant increase in the knee score and functional score after high tibial osteotomy for the patients of osteoarthritis with Varus deformity. The results are evident and maximal at 1 year after the procedure. No major complications occurred in patients with this procedure during the study.

Therefore, High tibial osteotomy should be recommended for the treatment of degenerative arthritis of the knee in young, active patients for symptomatic improvement and maintenance of activity levels.

\section{REFERENCES}

1. Catherine Hui, Lucy J Salmon, Alison Kok, Heidi A Williams, NielsHockers, Willem M. van der Tempel, Rishi Chana, Leo A. Pinczewski. Long-Term Survival of High Tibia Osteotomy for Medial Compartment Osteoarthritis of the Knee. The American Journa of sports medicin.2011;39(1):64-70.

2. Cooke TD. Definition of axial alignment of the lower extremity. J Bone Joint Surg Am 2002: $84 \cdot 146-147$

3. Virolainen P, Aro HT High tibial osteotomy for the treatment of osteoarthritis of the knee: a review of the literature and a meta-analysis of follow-up studies. Arch Orthop Trauma Surg 2004; 124:258-261.

4. Hernigou P, Medevielle D, Debeyre J. Goutallier D. Proximal tibial osteotomy for osteoarthritis with varus deformity. A ten to thirteen-year follow-up study. J Bone Joint Surg Am 1987; 69:332-354

5. Nakamura E, Mizuta H, Kudo S, Takagi K, Sakamoto K. Open-wedge osteotomy of the proximal tibia with hemicallotasis. The Journal of Bone and Joint Surgery 2001; 83(8):1111-5.

6. Meding JB, Kearing EM, Ritter MA et al. Total knee arthroplasty after high tibia osteotomy. Clinorthop. $2000 ;(375): 175-84$

Goutallier D, Stephane Van, Driessche SV, Manicom O, Ali ES, Bernageau J, et al. Influence of lower-limb torsion on long-term outcomes of tibial valgus osteotomy for medial compartment knee osteoarthritis. J Bone Joint Surg Br 2006; 88:2439-47.

8. Bonasia DE, Dettoni F, Sito G, et al. Medial opening wedge high tibial osteotomy for medial compartment overload/ arthritis in the varus knee: prognostic factors. Am J Sports Med. 2014; 42:690-698

9. Hawker GA, Wright JG, Coyte PC, Williams JI, Harvey B, Glazier R, et al. Differences 

2000; 342:1016-22.

10. Debeyre J, Patte D (1961) [The place of corrective osteotomies in the treatment of gonarthrosis.] Acta Orthop Belg; 27:374-383. French.

11. Aglietti P, Rinonapoli E, Stringa G, et al. Tibial osteotomy for the varus osteoarthritic knee. Clin Orthop Relat Res. 1983 ; (176):239-251

12. Flecher X, Parratte S, Aubaniac JM, et al. A 12-28-year follow up study of closing wedge high tibial osteotomy. Clin Orthop Relat Res. 2006; 452:91-6.

13. Howells NR, Salmon L, Waller A, et al. The outcome at ten years of lateral closingwedge high tibial osteotomy: determinants of survival and functional outcome. Bone Joint J. 2014; 96-B: 1491-1497.

14. Naudie D, Bourne RB, Rorabeck CH, Bourne TJ. The Install award: survivorship of the high tibial valgus osteotomy: A 10 to 22 years follow up study. Clin Orthop Relat Res 1999; 367:18-27.

15. Jung WH, Takeuchi R, Chun CW, Lee JS, Ha JH, Kim JH. Second-look arthroscopic assessment of cartilage regeneration after medial opening-wedge high tibial osteotomy. Arthroscopy 2014; 30:72-9.

16. Gennaro Pipino, Pier Francesco Indelli, Domenico Tigani, Giuseppe Maffei, Davide Vaccaris. Opening-wedge high tibial osteotomy: a seven - to twelve-year study: Joints. 2016 Jan-Mar; 4(1): 6-11.

17. Werner Kolbe MD, Hanno Guhlmann MD, Christoph Windisch MD, Heiko Koller MD, Paul Grützner MD, Klaus Kolb MD. Opening-Wedge High Tibial Osteotomy with a Locked Low-Profile Plate-Surgical Technique: JBJS 2009; 91-A(11):2581-2588. 